\title{
On-line Detection Method of Quality Defects in Manufacturing Process based on Wavelet Finite Element
}

\author{
Bin Zeng ${ }^{1, a}$, Yuxi Wang ${ }^{2, b^{*}}$, Quanming $\mathrm{He}^{3, \mathrm{c}}$ and Xiaoli Jiang ${ }^{4, \mathrm{~d}}$ \\ ${ }^{1}$ School of Art Design, Humanities \& Tea Culture, Zhejiang A \& F University, Lin'an, Zhejiang, China \\ ${ }^{2}$ Jiyang College of Zhejiang A \& F University, Zhuji, Zhejiang, China \\ ${ }^{3}$ Lin'an Longyan Information Engineering Co., Ltd., Lin'an, Zhejiang , China \\ ${ }^{4}$ School of Forestry and Bio-technology, Zhejiang A \& F University, Lin'an, Zhejiang, China \\ azhengbing1976@126.com, b112306207@qq.com, czb@microgrid.com.cn, d21543896@qq.com
}

\section{Keywords: Lifting Wavelet; Adaptive Finite Element; Modal Parameters; Stress Wave}

\begin{abstract}
In this paper, researchers use a wavelet-based solution to enhance the adaptive finite element method flaw inherent frequency beam, and study to conclude a particular state and the intrinsic link between the modal parameters, and establish a precise qualitative and quantitative identification based on the establishment of internal defects lifting wavelet adaptive format FEM model, accurate identification of internal defects in the category, location, size and other qualitative and quantitative. Defecting detection technology will be applied to the adaptive wavelet lifting scheme based on the finite element method quality manufacturing process and have achieved good experimental results.
\end{abstract}

\section{Introduction}

In 1992, American R. J. ROSS and some others stress wave detection technology to identify the plate, in 1997[1], and some others stress wave 144 0.025x0.038xO.508m stakes (U.S. southern pine made) were detected, and measured data regression analysis, draw the correlation between stress wave propagation velocity and the degree of decay stakes [2]. Some others studied the application of vibration stimulus-response detection technology to detect preservative treated wood[3]. Literature studied the dynamic characteristics of timber damage location detection method will be applied based on vibration[4]. Yang Huimin and some others changes the attenuation of ultrasonic energy frequency components characterization methods timber defect information, by using a variety of defects in the wood pores comparative study to determine the spectral analysis method variation of pore size of defects and holes number, but its location on the distinction between obvious defects[5]. M. E. Tiitta and some others studied the application of Bayesian methods, KNN and the neural network classifier to analyze the sound - ultrasonic (AU) to detect the validity of decaying wood[6]. In literature, modal analysis of finite element method will be introduced into the timber in the past, FFT through experimental modal analysis results fit, and this finite element simulation method provides the feasibility of the application in the detection of defects in wood material theory[7]. Literature the composition and distribution of the different technologies in terms of detection of stress waves inside the timber spectral analysis [8]. Literature designed a timber-based non-destructive testing Labview virtual instrument system[9]. Literature using wavelet packet decomposition can accurately detect the ultrasonic signal into different frequency band range, andn extract an amount of change of energy in different frequency bands [10]. Test shows the amount of change of the signal energy in a defective specimen has significantly different specific band [11], and the next step for the application of pattern recognition methods, such as neural networks to identify the defect type specimen, provides an ideal feature vector[12].

In the abovel detection of relevant research literature, this paper mainly detects the wood outline, the current domestic and international timber carrying wooden material defect identification type of defect 
detection, and defect location cannot be achieved with the quantitative detection. While carrying wood materials and quantitative detection of defect location, they cannot achieve the type of defect detection, which not only affects the efficiency of detection, and detection of wasted resources, but more important is that the development and promotion of industrialization are not conducive to the practical application of research results and the actual product.

\section{On-line Detection Method of Quality Defects in Manufacturing Process based on Wavelet Finite Element}

This paper introduces the leading precision of the real small defects recognition methods[13], in order to detect defects in timber identification, introduce some ways to solve the problem of inappropriate, the use of leading technology[14] to upgrade its technology architecture, and cutting-edge research methods currently testing field[15] combining domestic timber, the identification methods used in the manufacturing process of wood processing line detection.

(1). Structural identification unit based on lifting scheme wavelet defects

Specific steps:

Consider timber from the perspective of internal defects caused by linear elastic fracture mechanics topical additional flexibility to construct wavelet defect identification unit. References [16]the method introduces the method which was constructed by Sweldens[17] in 1996 to enhance the approach adopted in the time domain to construct a class of wavelet(Lifting Scheme Wavelet Transform), structure based on wavelet lifting scheme wavelet thin curved beam element and unit.

The similar literature[17] can be introduced on the basis of the lifting scheme wavelet analysis in the literature [15], this paper proposed an algorithm to obtain a second generation wavelet space element stiffness matrix $\tilde{k}_{e}, \tilde{k}_{e}$ With the conversion formula $k_{e}=T^{T} \tilde{k}_{e} T$ converted into physical space[14]. Then using the above method to construct the other scale lifting scheme wavelet unit, lifting scheme wavelet finite element thus researchers obtained with conventional finite element calculation has the same format.

(2). Adaptive wavelet lifting scheme based on finite element calculation upgrade

Specific steps:

1) Constructed on the basis of the lifting scheme wavelet defect recognition unit on the natural frequency of the beam is introduced to solve the defects based on lifting wavelet adaptive finite element method format, According to the calculation in response to the defect before the three natural frequencies[15], for solving the dynamic characteristics of the wood beams of internal defects, defects in the beam to get the essence of a sign on its first three natural frequencies reflect, get the essence of signs defects beam on its first three natural frequencies reflect.

ii) Based on Laplace wavelet-based modal precise identification [16] observed defect analysis cuts through the excitation frequency response characteristics of the measured beam defects, defects cantilever beam to obtain the first three natural frequencies ( $1, \mathrm{f} 2, \mathrm{f} 3$ )

iii) Enhance the adaptive coupling calculation: References [16, 17 theory, based on the references [17] proposed an adaptive algorithm to complete the upgrade to enhance the adaptive coupling calculation.

Which enhance the adaptive coupling calculation process $\mathrm{C} 0$ unit finite element solution Fang Chenggang matrix decoupling conditions for:

By introducing the proposed two vanishing moments stiffness matrix $K^{e}$ decoupling conditions: low-resolution approximation space and detail the space between coupling term $K_{\phi, \psi}^{0, k} 、 K_{\psi, \phi}^{k, 0}$; coupling terms between different spatial scales details $K_{\psi, \psi}^{m, k}(m \neq k)$ 
By constructing non-vanishing moments properties interpolating wavelet

(3). Intelligent classification of various defects

As a basis functions to construct a finite element approximation space process, wavelet enhances the low-resolution spatial resolution by increasing the detail of space to space, but there are coupling units in the space between the low-resolution approximation and detail details different scales of space and the space of the coupling term. Above conditions can eliminate the coupling term decoupling, smoothing approach to achieve the separation and detail information.

Enhance the adaptive coupling calculation process, lifting scheme wavelet adaptive finite element analysis model can be created directly obtain the corresponding fi $(\mathrm{i}=1,2,3)$ of the three equivalent stiffness on the relative position of the curve, the position of internal defects diagnosed timber, depth. Fig. 1 can be determined by its intersection A timber relative position of internal defects and defect equivalent stiffness (Corresponds to the relative geometry - Depth)

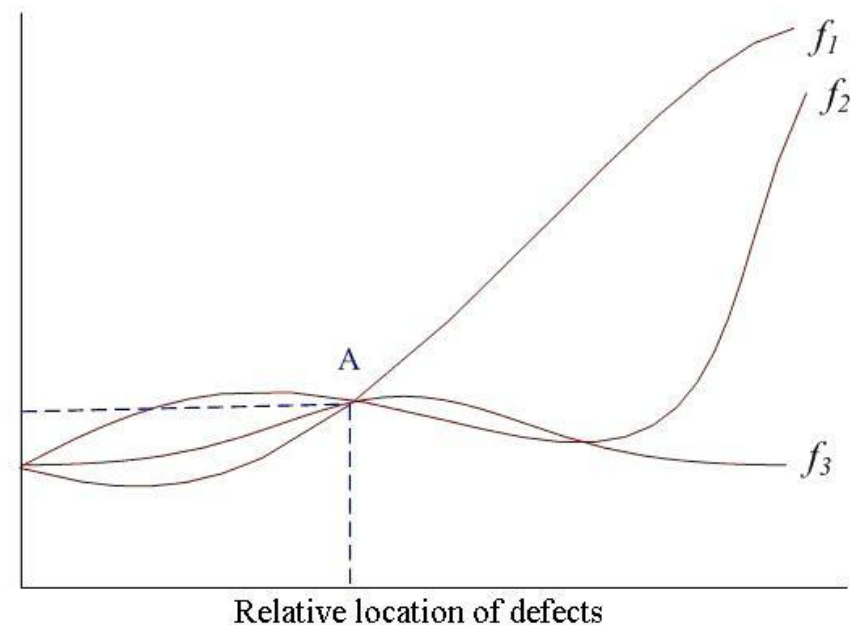

Relative location of defects

Fig. 1 Calculated to Enhance the Adaptive Process Coupled Three-line Diagnosis Wood Interior Defect Location, Depth

However, according to the above process diagnostics to determine the relative position of the timber internal defects and defect equivalent stiffness, equivalent stiffness corresponding defect relative geometry only and the total height of the depth of the measured component parallel to the beam( literature [15]), the depth of one-dimensional feature quantity inside the timber does not reflect the particular geometry of the three-dimensional defects, it is necessary to solve this problem while adding the identification of various types of defects.

This paper appoints its own lifting scheme wavelet transform (second generation wavelet transform) based on analysis of changes in the energy spread function defects, in order to enhance the adaptive coupling calculation process, the mutant signal decomposition, reconstruction and de-noising, identify the type of defect energy change according to the defect, and build defect propagation energy changes associated with the defect type mathematical model. For a variety of defects using neural network classification algorithm intelligence

\section{Summary}

In the same experimental conditions described herein format based on lifting wavelet adaptive finite element method and the literature's methods[8] with the literature $[2,3,10]$ the method is verified by comparing this study with leading technology and efficiency[13]. In the actual processing of timber production process, the test results and the real and artificial logs or timber internal section comparison validate that the method has 
good accuracy and validity. In the actual wood processing of enterprises using logs, timber, plywood, specimen trees and field tested in vivo binding complex from a more realistic situation, the greater the level of testing the validity and completeness of the theory, and further narrow the theory and practical application, the product distance. In summary, the use of the line defect detection technique based on lifting scheme wavelet adaptive finite element method to the actual quality of the wood timber manufacturing process, achieved good experimental results.

\section{Acknowledgement}

This work was supported by Zhejiang Science and Technology Department Project, No.2012C21087, 2014C32G2100058, 2013R30003.

\section{References}

[1] Xiaodong Zhu, Fenghu Wang, Jianping Sun, Virtual Instrument in non-destructive testing of timber. Key Laboratory for Materials Science and Technology, Ministry of Education, Northeast Forestry University, 2006

[2] Lihai Wang, Huimin Yang, Quantitative Detection of ultrasonic wavelet and neural network holes in wood color defects. Journal of Beijing Forestry University, 2007, 29(2):128-132

[3] Wei Qi, Lihai Wang, Wavelet packet analysis in ultrasonic testing of timber, Forest Engineering 2005, 21(3):24-25, 39

[4] XinFang Duan, Yudong Li,Ping Wang. NDT technology in the protection of timber. Timber Industry, 2002, 16(5):14-16

[5] Senming Guo, Status and Development of particleboard production line testing techniques, Plywood Communications, 2004(8), 8-10

[6] Hongjian Zhang, QiChen, Tianguo Wen, Kunyan Bao, Jianjun Zhang, Zhaoming Liao, Mechanically-Panel Quality Online destructive testing technology development and application, Forest Products Industry, 2007(6), 33-35

[7] Shanqing Liang, Zhiyong Cai, Xiping Wang, Robert J1 ROSS2, Feng Fu, North American wood nondestructive testing technology research and application, Timber Industry, 2008.5, 3(22), 5-8

[8] Huadong Xu, Lihai Wang, Songyuan Ni, Modal analysis in different locations in the hole defect detection plate. Forestry Machinery \& Woodworking Equipment, 2007, 35(11):47-49

[9] Huadong Xu, Lihai Wang, Songyuan Ni, Progress in the Application of Wood Properties detection modal analysis technique, Forest Engineering, 2007,23(6):15-17

[10] Chang Su, Yutuo Chen, Yunshui Yu, Xiaoyun Zhang, Wood defect detection based on wavelet transform and mathematical morphology, Computer Engineering and plications, 2008, 4(33):246-248

[11] Xidong Zhang, Lihai Wang, Xuechun Yang, Research on the impact of conditions on defect detection hole logs the Effect, Forestry Science and Technology, 2009,34(3):46-48

[12] Lihai Wang, Huadong Xu, Zaixing Yan, Jianxiong Lv, Xuechun Yang, Cilin Zhou, Affect the quantity and distribution of the stress wave sensors to detect the effect of wood defects, Forestry Science, 2008,44(5):115-121 
[13] Wei Qi, Lihai Wang, Based on the type of research to identify defects in the internal timber WNN, Forestry Science, 2006, 42(8):63-68

[14] Sici Yin, Wood Science[M]. beijing: China Forestry Publishing House, 1996: 184-213

[15] Zhengjia He, Yanyang Zeng, Laplace Wavelet its engineering application, Journal of Engineering Mathematics, 2001,18(5):87-92

[16] Lele S P, Maiti S K. Modelling of transverse vibration of short beams for crack detection and measurement of crack extension. Journal of Sound and Vibration, 2002, 257(3): 559-583

[17] KardestUncerH, Finite Element Method Manual (Dechao Zhu,Zizhi Fu translation) Beijing: Science Press, 1996 